\title{
EDITORIAL
}

\section{Combining information from prognostic scoring tools for CAP: an American view on how to get the best of} all worlds

\author{
M.S. Niederman*, C. Feldman ${ }^{\#}$ and G.A. Richards ${ }^{\#}$
}

$\mathbf{M}$ any studies of the epidemiology of patients with community-acquired pneumonia (CAP) have demonstrated the importance of assessing severity of illness and stratifying patients on the basis of their risk of mortality [1-3]. Optimal management of this illness requires prompt recognition of seriously ill patients to avoid mistakes such as the failure to use a hospital or intensive care unit (ICU) for patients who could benefit from care and observation in such settings. The major impact on the cost of CAP care is determined by whether or not a patient is admitted to the hospital. In the USA, $<20 \%$ of all CAP patients are admitted, but the number of dollars spent on these patients accounts for $>90 \%$ of the total cost of care for this disease [4].

Prognostic scoring systems for CAP have been developed to address these issues. The two prominent tools for this purpose are the Pneumonia Severity Index (PSI), developed in the USA, and the British Thoracic Society rule, which has recently been modified to the CURB-65 rule (referring to its assessment of: Confusion, elevated blood Urea nitrogen, elevated Respiratory rate, low systolic or diastolic Blood pressure, and age $>65$ yrs) $[1,3]$. Although each of the two approaches has been proposed as a tool to guide the site of care decision, neither is ideal by itself, and both can be regarded only as providing decision support information that must be supplemented by clinical assessment and judgment. In fact, the two scoring approaches should be viewed as being complementary, as each has different strengths and weaknesses. The PSI seems to have been developed, and best validated, as a way to identify low mortality risk patients, but the scoring system can occasionally underestimate severity of illness, especially in young patients without comorbid illness $[2,5]$. This is primarily because the PSI heavily weights age and comorbidity, and does not directly measure CAP-specific disease severity. In contrast, the CURB65 approach may be ideal for identifying high mortality risk patients with severe illness due to CAP who might otherwise be overlooked without formal assessment of subtle aberrations in key vital signs [3]. However, one clear deficiency of the

\footnotetext{
*Dept of Medicine, Winthrop University Hospital, Mineola, NY, USA. \#Division of Pulmonology, Dept of Medicine, Johannesburg Hospital, University of Wittswatersrand, Johannesburg, South Africa.

CORRESPONDENCE: M.S. Niederman, Winthrop University Hospital, 222 Station Plaza N., Suite 509I, Mineola, NY 11501, USA. Fax: 1 5166638796. E-mail: mniederman@winthrop.org
}

CURB-65 approach is that it does not generally account for comorbid illness, and thus may not be easily applied in older patients who may still have substantial mortality risk, even if a mild form of CAP destabilises a chronic, but compensated, disease process. Thus, both tools offer a valuable assessment of patient illness, but from different perspectives, and each is best at identifying patients at opposite ends of the disease severity spectrum.

In the current issue of the European Respiratory Journal, CAPELASTEGUI $e t$ al. [6] have used both the PSI and the CURB65 approach to evaluate a large number of inpatients and outpatients with CAP. They observed that the CURB-65 (and its simpler CRB-65 (Confusion, elevated Respiratory rate, low systolic or diastolic Blood pressure, and age $>65$ yrs) version, which excludes measurement of blood urea nitrogen, and therefore can be used in outpatients) can accurately predict 30day mortality, the need for mechanical ventilation and, to some extent, the need for hospitalisation. In addition, the CURB-65 criteria correlated with the time to clinical stability and thus a higher score was predictive of a longer duration of intravenous therapy and a longer length of hospital stay. The PSI also worked well to predict mortality, but, while other studies have established that the PSI was not good for predicting the need for ICU admission, CAPELASTEGU et al. [6] found that the CURB-65 was also not good for this purpose [5]. This conclusion has not been seen in all previous studies; some have shown that the CURB-65 is more accurate than the PSI for predicting the need for ICU admission. However, it did have limited positive predictive value [2]. One reason for the CURB65 having had limited value for predicting ICU admission in the study by CAPELASTEGUI $e t$ al. [6] was the fact that very few patients (maybe inappropriately) were admitted to the ICU. Other limitations in the study were the fact that some terminally ill patients were included, the scoring system was not used to prospectively determine the site of care decision, and the therapy for severe CAP was often suboptimal, allowing for the use of monotherapy with either a quinolone or $\beta$-lactam, both of which may increase mortality in patients with severe CAP $[7,8]$.

There have been other studies that have evaluated the PSI and some that have compared this tool directly to the CURB65 criteria. One major advantage of the CURB-65 approach to the PSI may be its simplicity in calcuation, but in order to promote simplicity, this scoring system ignores the important 
impact of comorbid illness on disease assessment, prognosis and management. For example, in the USA, in a large casecontrol study of CAP in 623,718 Medicare recipients, the overall mortality rate was $10.6 \%$, but rose higher with advancing age, nursing home residence and comorbid illness [9]. In fact, in this population, mortality risk varied widely based on a number of the factors included in the PSI, but not in the CURB-65 criteria, which could not be so discriminating in this population, since all patients were generally aged $\geqslant 65$ yrs. Thus, although the CURB-65 may be more of a direct measurement of disease severity than the PSI, it does not consider the presence of comorbid illness in predicting CAP outcome.

The PSI, however, is heavily influenced by both age and comorbidity, but is not really a measure of disease severity. For example, a low PSI score is possible in severe illness, especially in a young patient without comorbidity who has vital sign abnormalities that fall just below the dichotomous thresholds of the scoring system (such as a patient with a respiratory rate of 29 breaths $\cdot \mathrm{min}^{-1}$ ), a blood pressure of $92 / 50$, and a heart rate of 120 beats $\left.\cdot \mathrm{min}^{-1}\right)$. In fact, some studies have reported that $27-37 \%$ of all patients admitted to the ICU have been in PSI classes I-III [2, 5]. In these studies, while the PSI has consistently been a good predictor of mortality, the findings make it clear that risk of death may not be the same as need for hospital admission or ICU care.

In one recent study that compared the PSI with CURB-65, both were good for predicting mortality and identifying low mortality risk patients [10]. However, the PSI appeared to be more discriminating in identifying the low mortality risk patients. Using both approaches in 3,181 patients seen in an emergency department, $68 \%$ of patients were defined by PSI to be low risk (classes I-III) and had a mortality of $1.4 \%$, while $61 \%$ were low risk by the CURB-65 (score of $0-1$ ), with a mortality rate of $1.7 \%$. Thus, if the primary purpose is to avoid overestimating mortality risk, then the PSI may have a slight advantage. However, the CURB-65 may have been more valuable at the severe disease end of the spectrum because it defined high-risk patients as those with a score of 2, 3, 4 or 5. In the study, each of these classes had a progressively increasing risk of death, while the PSI was less discriminating, defining only two groups as being severely ill. Thus, the PSI class IV and $\mathrm{V}$ patients had mortality rates of 8.1 and $24 \%$, respectively, while the mortality rate in CURB-65 classes 3, 4 and 5 was 13, 17 and $43 \%$, respectively.

In another study, the CURB-65 score also appeared to identify, most accurately, those patients with CAP who were particularly likely to benefit from treatment with drotrecogin alpha in the Recombinant Human Activated Protein C Worldwide Evaluation in Severe Sepsis (PROWESS) study. A re-examination of the data from that study demonstrated that a threshold CURB-65 score of $\geqslant 3$ was associated more with a decrease in 28 -day mortality in drotrecogin alpha-treated patients of $10.8 \%$ when compared with controls $(p=0.018)$ versus a decrease in mortality in treated patients in PSI classes IV and V of $9.7 \%$ compared with controls $(p=0.013)$ [11]. The CURB-65 has also been studied in patients in South Africa with bacteraemic pneumococcal pneumonia, including both HIV-seropositive and -seronegative individuals, and was found to have a similar predictive ability for mortality to the PSI, even in HIVseropositive patients with a median CD4 cell count of $<200$ cells $\mu \mathrm{L}^{-1}[12]$.

Given the benefits and limitations of prognostic scoring systems in CAP, what is their real value? Clearly, both the PSI and CURB-65 are good for predicting mortality, but neither is ideal by itself, and neither can be used to define the site of care without considering other clinical and social variables. In particular, neither prognostic scoring system is able to measure "social factors" that add to the need for hospitalisation. One recent study at a public hospital in the USA, with many indigent patients, showed that the PSI could not define the need for admission if patients were homeless or acutely intoxicated, or if they did not have a stable home environment that allowed them to be discharged on oral antibiotic therapy [13]. Scoring systems can also have value in research and quality improvement to allow comparison of patient groups and to see if observed outcomes meet expected outcomes and are comparable among similar patients. In clinical research, it is unlikely that either the PSI or CURB-65 alone will be ideal, and one may be preferable to the other, depending on the purpose of the study.

Our preference is to combine both of these prognostic scoring tools, along with a clinical evaluation, to develop an algorithm for defining the site of care decision in community-acquired pneumonia patients, recognising that neither approach can stand alone. This algorithm would allow low-risk patients (Pneumonia Severity Index I-III or CURB-65 of $0-1$ ) to be managed at home if serious vital sign abnormalities (in the case of Pneumonia Severity Index) or comorbidities (in the case of CURB-65) are absent, and if patients do not have social factors or other illnesses that are unstable and that necessitate hospitalisation. Moderate risk patients (CURB-65 of $\geqslant 2$, or Pneumonia Severity Index classes IV and V) would be admitted, and scoring systems and clinical assessments could be used to separate those who need intensive care unit care from those who are likely to become clinically stable rapidly and who would then require only a short hospital stay. It is likely that a prospective study that included such an algorithm could document the value of either scoring approach, but it is also likely that combining the two would be the best idea, since each is different and complementary to the other, with each serving to best identify patients at opposite ends of the disease severity spectrum.

\section{REFERENCES}

1 Fine MJ, Auble TE, Yealy DM, et al. A prediction rule to identify low-risk patients with community-acquired pneumonia. N Engl J Med 1997; 336: 243-250.

2 Ewig S, De roux A, Bauer T, et al. Validation of predictive rules and indices of severity for community acquired pneumonia. Thorax 2004; 59: 421-427.

3 Lim WS, van der Erden MM, Laing R, et al. Defining community acquired pneumonia severity on presentation to hospital: an international derivation and validation study. Thorax 2003; 58: 377-382.

4 Niederman MS, McCombs JS, Unger AN, Kumar A, Popovian R. The cost of treating community-acquired pneumonia. Clin Ther 1998; 20: 820-837. 
5 Angus DC, Marrie TJ, Obrosky S, et al. Severe communityacquired pneumonia: use of intensive care services and evaluation of the American and British Thoracic Society criteria. Am J Respir Crit Care Med 2002; 166: 717-723.

6 Capelastegui A, España PP, Quintana JM, et al. Validation of a predictive rule for the management of communityacquired pneumonia. Eur Resp J 2006; 27: 151-157.

7 Brown RB, Iannini P, Gross P, Kunkel M. Impact of initial antibiotic choice on clinical outcomes in communityacquired pneumonia: analysis of a hospital claims-made database. Chest 2003; 123: 1503-1511.

8 Leroy O, Saux P, Bedos JP, Caulin E. Comparison of levofloxacin and cefotaxime combined with ofloxacin for ICU patients with community-acquired pneumonia who do not require vasopressors. Chest 2005; 128: 172-183.

9 Kaplan V, Angus DC, Griffin MF, Clermont G, Scott Watson R, Linde-Zwirble WT. Hospitalized community-acquired pneumonia in the elderly: age- and sex-related patterns of care and outcome in the United States. Am J Respir Crit Care Med 2002; 165: 766-772.

10 Aujesky D, Auble TE, Yealy DM, et al. Prospective comparison of three validated prediction rules for prognosis in community-acquired pneumonia. Am J Med 2005; 118: 384-392.

11 Laterre PF, Garber G, Levy H, et al. Severe communityacquired pneumonia as a cause of severe sepsis: data from the PROWESS study. Crit Care Med 2005; 33: 952-961.

12 Feldman C, Richards G, Klugman KP, Yu V. CURB-65 clinical prediction rule in invasive pneumococcal disease (IPD). Eur Respir J 2005; 26: 663S.

13 Goss CH, Rubenfeld GD, Park DR, Sherbin VL, Goodman MS, Root RK. Cost and incidence of social comorbidities in low-risk patients with communityacquired pneumonia admitted to a public hospital. Chest 2003; 124: 2148-2155. 